\title{
SALUD DE MIGRANTES INTERNACIONALES EN SITUACIÓN IRREGULAR: UNA REVISIÓN NARRATIVA DE INICIATIVAS
}

\author{
Daniel Larenas-Rosa ${ }^{1,2, a}$, Báltica Cabieses Valdés ${ }^{1,3, b}$
}

\begin{abstract}
RESUMEN
La población migrante internacional en situación irregular (MISI) suele encontrarse en una condición de particular vulnerabilidad que impacta en su salud, y sumado a esto, enfrenta múltiples barreras que dificultan e incluso impiden su acceso a los servicios de salud. La presente revisión tiene por objetivo identificar y describir iniciativas que abordan el acceso a los servicios de salud en población MISI. Para ello, se realizó una revisión narrativa en PubMed, Embase, Health Systems Evidence, Cochrane, LILACS y Scielo entre julio y agosto de 2017. Se encontraron 787 artículos, de los cuales se incluyeron 26, los que dieron cuenta de 31 iniciativas. La mayoría de estas iniciativas se sitúan en América Septentrional (61\%), Asia Sudoriental (10\%) y Europa Septentrional (10\%); se refieren mayoritariamente a servicios de nivel primario $(48 \%)$ y terciario $(52 \%)$, servicios de urgencia/emergencia $(39 \%)$, brindan accesibilidad administrativa $(65 \%)$ y financiera (45\%), y responden a necesidades en la etapa de «destino» del proceso migratorio (97\%). Se puede concluir que existen diversas iniciativas que abordan la problemática del acceso a los servicios de salud de la población MISI. No obstante, no se hallaron iniciativas dirigidas a la rehabilitación y salud sexual; contacto y calidad; perspectiva de género y competencia cultural en salud.
\end{abstract}

Palabras clave: Migrantes en situación irregular; Migración internacional; Emigrantes e inmigrantes; Acceso a los servicios de salud; Política de salud (fuente: DeCS BIREME).

\section{HEALTH OF INTERNATIONAL MIGRANTS IN AN IRREGULAR SITUATION: A NARRATIVE REVIEW OF INITIATIVES}

\begin{abstract}
The population of international migrants in an irregular situation (IMIS) often finds itself in a condition of particular vulnerability that has an impact on its health. Additionally to this, these migrants face multiple barriers that hinder or even impede their access to health services. This review aims at identifying and describing initiatives that address access to health services in the IMIS population. To this end, a narrative review was conducted in PubMed, Embase, Health Systems Evidence, Cochrane, LILACS, and Scielo, between July and August of 2017. Seven hundred and eighty-seven (787) articles were found, of which 26 have been included, accounting for 31 initiatives. Most of these initiatives are located in North America (61\%), South-East Asia (10\%), and Northern Europe (10\%). They refer mostly to primary (48\%) and tertiary (52\%) services; urgency/emergency services (39\%); provide administrative $(65 \%)$ and financial accessibility $(45 \%)$; and respond to needs at the "destination" stage of the migration process $(97 \%)$. It can be concluded that there are several initiatives that deal with the problems faced by the IMIS population in terms of access to healthcare services. However, no initiatives were found aimed at rehabilitation and sexual health; contact and quality, gender perspective, and cultural competence in health.
\end{abstract}

Keywords: Undocumented immigrants; International migration; Emigrants and immigrants; Access to health care; Health policy (source: MeSH NLM).

\footnotetext{
1 Programa de Estudios Sociales en Salud, Instituto de Ciencias e Innovación en Medicina, Facultad de Medicina Clínica Alemana, Universidad del Desarrollo. Santiago, Chile.

2 Departamento de Fonoaudiología, Facultad de Medicina, Universidad de Chile. Santiago, Chile.

3 Department of Health Sciences, University of York. England, United Kingdom.

a Fonoaudiólogo, magíster en Salud Pública; ${ }^{b}$ enfermera matrona, $\mathrm{PhD}$ en Epidemiología Social Recibido: 17/04/2019 Aprobado: 31/07/2019 En línea: 16/08/2019
}

Citar como: Larenas-Rosa D, Cabieses Valdés B. Salud de migrantes internacionales en situación irregular: una revisión narrativa de iniciativas. Rev Peru Med Exp Salud Publica. 2019;36(3):487-96. doi:http://dx.doi.org/10.17843/rpmesp.2019.363.4469. 


\section{INTRODUCCIÓN}

La migración internacional aumentó durante las últimas dos décadas a nivel mundial. Se estima que en el 2000 existían entre 155 y 173 millones de migrantes internacionales, llegando a 258 millones en 2017 (3,4\% de la población mundial total) ${ }^{(1,2)}$. Un grupo que suscita particular interés es la población migrante internacional en situación irregular (MISI). En 2008 se estimó que entre 1,9 y 3,8 millones de migrantes se encontraban en situación irregular en la Unión Europea ${ }^{(3)}$; mientras que en Estados Unidos en 2012 la población MISI correspondía a 11,4 millones ${ }^{(4)}$. Según la Comisión Interamericana de Derechos Humanos, se denomina migrante internacional en situación irregular a quien ingresa al territorio de un Estado en forma irregular o que permanece en él por más tiempo del que se le autorizó ${ }^{(5)}$.

Muchos MISI requieren protección producto de catástrofes naturales y crisis sociales y económicas en sus países de origen, o ya que durante el tránsito pueden ser víctimas de trata, trauma y/o violencia ${ }^{(6)}$. En el país de destino suelen desempeñarse en trabajos de baja remuneración, carecen de redes de apoyo, enfrentan situaciones de separación familiar y presentan signos de trauma, depresión y estrés crónico ${ }^{(7)}$. Su vulnerabilidad y marginalización impacta en su salud y, al mismo tiempo, carecen de acceso a los servicios de salud ${ }^{(8)}$.

La Convención internacional sobre la protección de los derechos de todos los trabajadores migratorios y de sus familias, explicita el derecho a recibir atención médica urgente en las mismas condiciones que los nacionales, la que no puede ser negada a causa de irregularidad ${ }^{(9)}$. En el 2000, el Comité de derechos económicos, sociales y culturales señalaba la obligación de los Estados de respetar el derecho a la salud y de abstenerse de denegar o limitar el acceso a la población MISI a servicios preventivos, curativos y paliativos ${ }^{(10)}$.

Pese a lo anterior, en la mayoría de los países existen múltiples barreras que hacen a las normativas inefectivas. Parte de la población MISI no asiste a los centros de salud por temor a los sistemas de registro y a su asociación con la deportación ${ }^{(11,12)}$. Asimismo, se enfrentan al desconocimiento sobre el acceso al sistema de salud y a las barreras lingüísticas y comunicativas ${ }^{(13)}$. Se han documentado barreras de tipo interpersonal, donde funcionarios y equipos de salud ejercen actos discriminatorios por aumento de los costos, falta de cupos o razones discrecionales como el país de origen o el color de la piel (14-17).

La relevancia de implementar medidas que permitan garantizar el acceso a la atención de salud a la población MISI es reconocida mundialmente. El Consenso de Montevideo sobre Población y Desarrollo, y una resolución del Parlamento Europeo del 2011 son instancias en las que se acordó garantizar el acceso a la asistencia sanitaria a la población MISI ${ }^{(18,19)}$. Pese a esta importancia, en la actualidad no se han reportado revisiones que den cuenta de las medidas implementadas para abordar el acceso a servicios de salud en esta población. El propósito de la presente revisión narrativa es identificar y describir iniciativas gubernamentales y no gubernamentales que den respuesta a la problemática del acceso a los servicios de salud que enfrenta la población MISI, que hayan sido implementadas o que son potencialmente implementables a nivel federal, estatal o local.

\section{BÚSQUEDA Y SELECCIÓN DE ARTÍCULOS}

\section{BÚSQUEDA DE ARTÍCULOS}

La búsqueda se realizó en las bases de datos PubMed, Embase, Health Systems Evidence, Cochrane, LILACS y Scielo entre julio y agosto del 2017. La estrategia de búsqueda se detalla en el Anexo 1.

\section{SELECCIÓN DE ARTÍCULOS}

Tras obtener los resultados de búsqueda, se eliminaron las repeticiones. Para la selección de los artículos se examinaron los títulos y resúmenes. Como criterios de inclusión se estableció que los estudios consideraran: 1) migrantes en situación irregular, 2) acceso/uso de servicios de salud e 3) iniciativas de salud a nivel federal, estatal o local. Se aceptaron artículos en idioma español o inglés y sin restricción de tiempo. Los estudios seleccionados se recuperaron en texto completo, se corroboraron los criterios de inclusión, quedando finalmente para la extracción y análisis de información.

\section{EXTRACCIÓN DE INFORMACIÓN Y ANÁLISIS}

La extracción de datos de los artículos seleccionados fue realizada mediante una matriz diseñada en Microsoft Excel que incluyó: nombre de la iniciativa, autor del estudio y año de publicación. Además, la matriz incluyó tres categorías de análisis: 1) contexto, que contempla las variables subregión y país en que se diseñó o implementó la iniciativa; 2) componentes del prestador, que contiene las dimensiones de disponibilidad, accesibilidad, aceptabilidad, contacto y calidad; y 3) características de la población, que comprende las dimensiones de etapa del proceso migratorio, etapa del ciclo vital, sistemas de creencias y género.

\section{SELECCIÓN DE ARTÍCULOS}

La búsqueda reportó 816 artículos, tras eliminar las repeticiones quedaron 787 artículos. Luego de examinar los títulos y resúmenes se seleccionaron 58 estudios. Finalmente, tras recuperar los textos completos y corroborar los criterios de inclusión se consideraron 26 estudios (Figura 1). A partir de los 26 estudios se identificaron 31 iniciativas, las cuales se describen en la Tabla 1. 

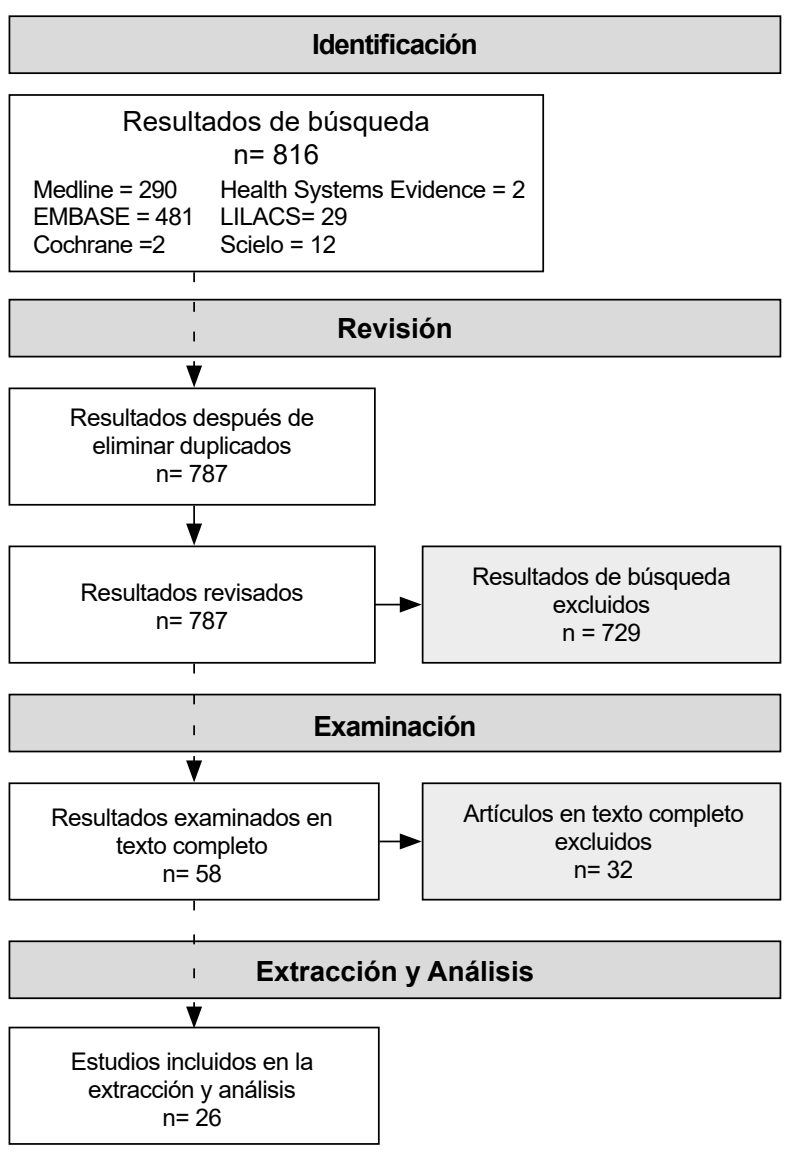

Figura 1. Flujograma de selección de artículos

Cada iniciativa fue clasificada geográficamente, de acuerdo a la clasificación de las Naciones Unidas para el uso estadístico M49 ${ }^{(20)}$. Mayoritariamente, se observan iniciativas en América Septentrional, específicamente 19 en Estados Unidos, seguida por Asia Sudoriental y Europa Septentrional, con tres iniciativas en cada una. En la Tabla 2 se presenta una síntesis de resultados globales obtenidos por cada variable según las dimensiones de análisis en el total de iniciativas estudiadas.

\section{CARACTERÍSTICAS DEL PRESTADOR DE SERVICIOS}

\section{DISPONIBILIDAD}

Seis iniciativas contemplaron acciones a nivel de la autoridad sanitaria, 15 contemplaron acciones de nivel primario, 12 de nivel secundario y 16 de nivel terciario. Las prestaciones más frecuentes fueron servicios de emergencia o urgencia en 12 iniciativas, servicios de especialidad médica en siete iniciativas, acciones de prevención en cinco iniciativas, acciones de salud comunitaria en cuatro iniciativas y chequeo médico en cuatro iniciativas (Tabla 3). No se hallaron prestaciones de salud sexual y reproductiva ni de rehabilitación.

\section{ACCESIBILIDAD}

En cuanto a "accesibilidad geográfica», cuatro iniciativas aproximan los servicios a las comunidades migrantes. El programa de asistencia a personas con tuberculosis de Estambul contempló lugares estratégicos de tamizaje en centros clínicos cercanos a zonas de alta densidad de población migrante, visitas domiciliarias y contacto constante con los pacientes para incentivarlos a permanecer en la ciudad hasta el final de su tratamiento ${ }^{(21)}$. Además, el acceso ampliado a clínicas comunitarias de California incluye comunidades desatendidas para facilitar la atención a los MISI (22). Los servicios innovadores y amigables de Tailandia incluyen clínicas móviles para comunidades migrantes, servicios extendidos a los lugares de trabajo y educadores sanitarios voluntarios de las mismas comunidades (23). Mientras que las unidades de búsqueda y rescate de Arizona facilitan servicios médicos de emergencia en zonas fronterizas ${ }^{(24)}$.

Asimismo, 20 iniciativas brindan «accesibilidad administrativa» para población MISI. En Francia, el beneficio de asistencia médica es accesible para los MISI con más de tres años de residencia y con documentación que verifique su identidad y domicilio; sino pueden certificar el tiempo mínimo de residencia, se le brinda cobertura de atención de emergencia, atención ante enfermedades infectocontagiosas, atención a niños, mujeres embarazadas y atención al recién nacido ${ }^{25}$. En Italia, el código de «Extranjero Temporalmente Presente» permite ala población MISI identificarse en todos los servicios de salud y acceder a los servicios de urgencia y tratamientos esenciales ${ }^{(26)}$. Otra iniciativa es el programa para pacientes no elegibles de los centros de salud académicos de Nuevo México, en el cual funcionarios y profesionales de la salud reciben capacitación estandarizada en políticas de elegibilidad para minimizar potenciales situaciones de discriminación (27).

En términos de "accesibilidad financiera», se hallaron 14 iniciativas. Algunas ofrecen esquemas de cobertura o servicios específicos a bajo costo. Un ejemplo es el esquema de protección del riesgo financiero para migrantes no cubiertos por el seguro social de salud de Tailandia, que consta de un plan de prepago financiado por una prima anual equivalente 73 USD, que cubre servicios curativos integrales, servicios de prevención y de promoción (23). Otra iniciativa corresponde al programa de filipinos en el extranjero incluidos MISI, que contempla el pago de una prima anual de 55 USD; el afiliado debe pagar al utilizar un servicio fuera del país y luego recibe el reembolso del programa ${ }^{(28)}$.

Existen iniciativas que establecen "servicios gratuitos» para candidatos que cumplen requisitos de elegibilidad, mientras que otros establecen la gratuidad universal. Healthy San Francisco es un programa de la ciudad de San Francisco, que brinda acceso universal a la atención 
Tabla 1. Resumen de iniciativas que abordan la problemática de acceso y uso de servicios de salud que enfrentan migrantes internacionales en situación irregular, según país, autor del artículo y año de publicación

\begin{tabular}{|c|c|c|c|}
\hline Iniciativa & País & Autores & Año \\
\hline Protección del riesgo financiero & Tailandia & Tangcharoensathien V, Thwin AA, Patcharanarumol W. & 2017 \\
\hline Servicios amigables para el migrante & Tailandia & Tangcharoensathien V, Thwin AA, Patcharanarumol W. & 2017 \\
\hline $\begin{array}{l}\text { Reforma de salud con cobertura para } \\
\text { inmigrantes }\end{array}$ & $\begin{array}{l}\text { Estados Unidos } \\
\text { (Massachusetts) }\end{array}$ & Joseph TD. & 2016 \\
\hline $\begin{array}{l}\text { Asistencia Médica del Estado (Aide Médicale } \\
\text { de l'Etat.) }\end{array}$ & Francia & André JM, Azzedine F. & 2016 \\
\hline Programa de Filipinos en el Extranjero & Filipinas & Guinto RL, Curran UZ, Suphanchaimat R, Pocock NS. & 2016 \\
\hline Estrategia de Diálisis Ambulatoria & $\begin{array}{l}\text { Estados Unidos } \\
\quad \text { (California) }\end{array}$ & Rodriguez RA. & 2015 \\
\hline Estrategia de Diálisis de Emergencia & $\begin{array}{l}\text { Estados Unidos } \\
\quad \text { (Texas) }\end{array}$ & Rodriguez RA. & 2015 \\
\hline $\begin{array}{l}\text { Programa de Asistencia a la Tuberculosis de } \\
\text { Estambul }\end{array}$ & Turquía & Yasin Y, Biehl K, Erol M. & 2015 \\
\hline $\begin{array}{l}\text { Código para extranjeros temporalmente } \\
\text { presentes }\end{array}$ & Italia & De Vito E, de Waure C, Specchia ML, Ricciardi W. & 2015 \\
\hline $\begin{array}{l}\text { Proyecto de Ley SB 741: Cobertura de } \\
\text { transplante renal a no ciudadanos }\end{array}$ & $\begin{array}{l}\text { Estados Unidos } \\
\quad \text { (Illinois) }\end{array}$ & Ansell D, Pallok K, Guzman MD, Flores M, Oberholzer J. & 2015 \\
\hline $\begin{array}{l}\text { Resolución Conjunta 98: Grupo de trabajo } \\
\text { legislativo para el estudio de políticas de } \\
\text { transplantes }\end{array}$ & $\begin{array}{l}\text { Estados Unidos } \\
\quad \text { (Illinois) }\end{array}$ & Ansell D, Pallok K, Guzman MD, Flores M, Oberholzer J. & 2015 \\
\hline $\begin{array}{l}\text { Real Decreto 16/2012: Exclusión de los } \\
\text { inmigrantes en situación irregular. }\end{array}$ & España & $\begin{array}{l}\text { Pérez-Molina JA, Pulido F, Prous CA, Pedrol PD, Loyarte JAI, Rupérez } \\
\text { JL., Álvarez CM., Riera-Jaume M, Fernández PV. } \\
\text { Pérez-Molina JA, Pulido Ortega F. }\end{array}$ & $\begin{array}{l}2015 \\
2012\end{array}$ \\
\hline Política de repatriación médica & Estados Unidos & Young MJ, Lehmann LS. & 2014 \\
\hline $\begin{array}{l}\text { Estrategia binacional de protección social de la } \\
\text { salud de los migrantes }\end{array}$ & México & Arredondo López, A, Orozco Núñez E, Wallace S; Rodríguez MI. & 2013 \\
\hline $\begin{array}{l}\text { Programa para pacientes no elegibles en } \\
\text { Centros de Salud Académicos }\end{array}$ & $\begin{array}{l}\text { Estados Unidos } \\
\text { (Nuevo México) }\end{array}$ & Cacari Stone L, Steimel L, Vasquez-Guzman E, Kaufman A. & 2014 \\
\hline $\begin{array}{l}\text { Ley de protección al paciente y cuidado de } \\
\text { salud asequible PPACA } \\
\text { (Acceso exclusivo a atención de emergencia) }\end{array}$ & Estados Unidos & $\begin{array}{l}\text { Edward J. } \\
\text { Stimpson JP, Wilson FA, Su D. }\end{array}$ & $\begin{array}{l}2014 \\
2013\end{array}$ \\
\hline $\begin{array}{l}\text { Ley de Reconciliación de Responsabilidad } \\
\text { Personal y Oportunidad (PRWORA) }\end{array}$ & Estados Unidos & Gilcrist AR. & 2013 \\
\hline Seguro de salud binacional & $\begin{array}{l}\text { Estados Unidos } \\
\quad \text { (California) }\end{array}$ & Wallace SP, Rodriguez M, Padilla-Frausto I, Arredondo A, Orozco E. & 2013 \\
\hline Seguro obligatorio por el empleador & $\begin{array}{l}\text { Estados Unidos } \\
\text { (California) }\end{array}$ & Wallace SP, Rodriguez M, Padilla-Frausto I, Arredondo A, Orozco E. & 2013 \\
\hline Acceso ampliado a clínicas comunitarias & $\begin{array}{l}\text { Estados Unidos } \\
\text { (California) }\end{array}$ & Wallace SP, Rodriguez M, Padilla-Frausto I, Arredondo A, Orozco E. & 2013 \\
\hline Derecho a atención de urgencia gratuita & Dinamarca & Biswas D, Toebes B, Hjern A, Ascher H, Norredam M. & 2012 \\
\hline Derecho a servicios de emergencia & Suecia & Biswas D, Toebes B, Hjern A, Ascher H, Norredam M. & 2012 \\
\hline $\begin{array}{l}\text { Sistema de reembolso específico de la } \\
\text { atención médicamente necesaria }\end{array}$ & Países Bajos & $\begin{array}{l}\text { Biswas D, Toebes B, Hjern A, Ascher H, Norredam M. } \\
\text { Grit K, den Otter JJ, Spreij A. }\end{array}$ & $\begin{array}{l}2012 \\
2012\end{array}$ \\
\hline San Fracisco Healthy Kids & $\begin{array}{l}\text { Estados Unidos } \\
\text { (San Francisco) }\end{array}$ & Marrow HB. & 2012 \\
\hline Healthy San Francisco & $\begin{array}{l}\text { Estados Unidos } \\
\text { (San Francisco) }\end{array}$ & Marrow HB. & 2012 \\
\hline Acceso a servicios de emergencias & Reino Unido & Grit K., den Otter JJ, Spreij A. & 2012 \\
\hline $\begin{array}{l}\text { Cuidado de enfermedad renal en estado } \\
\text { terminal para inmigrantes indocumentados }\end{array}$ & Estados Unidos & Campbell G, Sanoff S, Rosner M. & 2010 \\
\hline $\begin{array}{l}\text { Unidades de búsqueda, trauma y rescate de la } \\
\text { patrulla fronteriza }\end{array}$ & $\begin{array}{l}\text { Estados Unidos } \\
\text { (Arizona) }\end{array}$ & Keim SM, Reiser F, Shetty S, Ranger-Moore J. & 2009 \\
\hline $\begin{array}{l}\text { Iniciativas para la Salud de los Niños }(\mathrm{CHI}) \text { : } \\
\text { Programa Niños Saludables }\end{array}$ & $\begin{array}{l}\text { Estados Unidos } \\
\text { (California) }\end{array}$ & Stevens GD, Rice K, Cousineau MR. & 2007 \\
\hline Ley de Reembolso de Salud de Inmigrantes & Estados Unidos & Health Policy Monitor & 2003 \\
\hline $\begin{array}{l}\text { Eliminación del Financiamiento Público de la } \\
\text { Atención Prenatal }\end{array}$ & $\begin{array}{l}\text { Estados Unidos } \\
\text { (California) }\end{array}$ & Kuiper H, Richwald GA, Rotblatt H, Asch S. & 1999 \\
\hline
\end{tabular}


Tabla 2. Síntesis de resultados globales obtenidos por cada variable observada según dimensión de análisis de iniciativas que abordan la problemática de acceso y uso de servicios de salud que enfrenta la población migrante internacional en situación irregular

\begin{tabular}{|c|c|c|}
\hline Categorías de respuesta & $\mathbf{n}$ & $\%$ \\
\hline \multicolumn{3}{|l|}{ Contexto } \\
\hline \multicolumn{3}{|l|}{ Subregión } \\
\hline América Septentrional & 19 & 62 \\
\hline América Central & 1 & 3 \\
\hline Asia Occidental & 1 & 3 \\
\hline Asia Sudoriental & 3 & 10 \\
\hline Europa Meridional & 2 & 6 \\
\hline Europa Occidental & 2 & 6 \\
\hline Europa Septentrional & 3 & 10 \\
\hline \multicolumn{3}{|l|}{ Componentes del Prestador } \\
\hline \multicolumn{3}{|l|}{ Disponibilidad } \\
\hline \multicolumn{3}{|l|}{ Nivel de atención sanitaria* } \\
\hline Autoridad sanitaria & 6 & 19 \\
\hline Terciaria & 16 & 52 \\
\hline Secundaria & 12 & 39 \\
\hline Primaria & 15 & 48 \\
\hline \multicolumn{3}{|l|}{ Tipo de prestación* } \\
\hline Urgencia/Emergencia & 12 & 39 \\
\hline Especialidad & 7 & 23 \\
\hline Inmunización & 1 & 3 \\
\hline Salud mental & 3 & 10 \\
\hline Salud sexual y reproductiva & 0 & 0 \\
\hline Salud materno infantil & 2 & 6 \\
\hline Salud dental & 2 & 6 \\
\hline Salud preventiva & 5 & 16 \\
\hline Salud comunitaria & 4 & 13 \\
\hline Promoción de la salud & 1 & 3 \\
\hline Chequeo médico & 4 & 13 \\
\hline Rehabilitación & 0 & 0 \\
\hline Medicamentos & 3 & 10 \\
\hline \multicolumn{3}{|l|}{ Accesibilidad $^{*}$} \\
\hline Geográfica & 4 & 13 \\
\hline Administrativa & 20 & 65 \\
\hline Financiera & 14 & 45 \\
\hline \multicolumn{3}{|l|}{ Aceptabilidad } \\
\hline Competencia cultural en salud & 1 & 3 \\
\hline Control del estatus de residencia & 5 & 16 \\
\hline Contacto & 0 & 0 \\
\hline Calidad & 0 & 0 \\
\hline \multicolumn{3}{|l|}{ Características de la Población } \\
\hline \multicolumn{3}{|l|}{ Etapa del proceso migratorio* } \\
\hline Origen & 0 & 0 \\
\hline Tránsito & 1 & 3 \\
\hline Destino & 30 & 97 \\
\hline Retorno & 2 & 6 \\
\hline \multicolumn{3}{|l|}{ Curso de vida } \\
\hline Niños, niñas y adolescentes & 2 & 6 \\
\hline Adultos & 4 & 13 \\
\hline Adultos mayores & 0 & 0 \\
\hline \multicolumn{3}{|l|}{ Género } \\
\hline Mujeres & 0 & 0 \\
\hline Hombres & 0 & 0 \\
\hline LGTBI & 0 & 0 \\
\hline Sistema de creencias & 0 & 0 \\
\hline
\end{tabular}

médica primaria a todo residente entre 18 y 65 años, cuyos ingresos se ubiquen por debajo de la línea de la pobreza según la definición federal, y que no califiquen para otras formas de cobertura pública ${ }^{(29)}$. Además, una normativa en Dinamarca estableció la gratuidad de la atención de urgencia para los MISI ${ }^{(30)}$.

En contraposición, otras iniciativas proponen «eliminar el financiamiento público». En California un estudio evaluó la implementación hipotética de una norma que elimina el financiamiento público de la atención prenatal para los MISI, concluyendo que la atención prenatal adecuada se reduciría entre un $50 \%$ a $90 \%$, lo que generaría ahorros anticipados. No obstante, como consecuencia de esta norma, aumentarían los gastos médicos y resultados adversos del embarazo, reduciendo los ahorros anticipados ${ }^{(31)}$.

\section{ACEPTABILIDAD}

Una iniciativa que incorpora acciones relacionadas a «competencia intercultural en salud» se refiere a la implementación de servicios innovadores y amigables con los migrantes en Tailandia, que contempla señaléticas e información bilingüe en instalaciones de salud ${ }^{(23)}$.

Finalmente, cinco iniciativas están insertas en contextos en que el "control del estado de residencia» se relaciona con la atención de salud. La Ley de Protección al Paciente y Cuidado de Salud Asequible (PPACA) de Estados Unidos excluyó a los MISI de la posibilidad de acceder a un seguro de salud, en el contexto de un sistema de salud con un estricto sistema de verificación del estatus de ciudadanía ${ }^{(32)}$. De forma similar, la regulación de Reino Unido establece la obligación de cerciorarse del estatus de residencia de todo paciente atendido en hospitales ${ }^{(33)}$; y la normativa danesa de migración señala que los MISI pueden solicitar atención médica al servicio de migración, resultando paradójico, ya que, dentro de sus funciones, este tiene que alertar a la policía sobre el paradero del solicitante ${ }^{(30)}$.

\section{CARACTERÍSTICAS DE LA POBLACIÓN}

\section{ETAPA DEL PROCESO MIGRATORIO}

La salud de los migrantes podría comprometerse en cualquiera de las etapas del ciclo migratorio: origen, tránsito, destino y retorno ${ }^{(34)}$. El $97 \%$ de las iniciativas estudiadas se sitúan en la etapa de «destino» (Tabla 4). Una de las iniciativas está dirigida a la población migrante «en tránsito», la cual corresponde a las unidades de trauma, búsqueda y rescate de Arizona, que brindan atención médica de emergencia en zonas de tránsito ${ }^{(24)}$.

Dos iniciativas contemplan acciones relacionadas con el «retorno». Una es la repatriación médica, discutida en Estados Unidos en un contexto de inexistencia de 
Tabla 3. Iniciativas que abordan la problemática de acceso y uso de servicios de salud que enfrentan migrantes internacionales en situación irregular descritas según variables de análisis asociadas a los componentes del prestador de servicios

\begin{tabular}{|c|c|c|c|c|c|c|c|}
\hline \multirow{3}{*}{ Iniciativas $(n=31)$} & \multicolumn{7}{|c|}{ Características del prestador de servicios } \\
\hline & \multicolumn{2}{|c|}{ Disponibilidad } & \multicolumn{3}{|c|}{ Accesibilidad } & \multicolumn{2}{|c|}{ Aceptabilidad } \\
\hline & Nivel de atención & Tipo de prestación & G & A & $\mathbf{F}$ & CC & CM \\
\hline Protección del riesgo financiero & $\begin{array}{l}\text { Primario } \\
\text { Secundario }\end{array}$ & $\begin{array}{l}\text { Prevención } \\
\text { Promoción } \\
\text { Medicamentos }\end{array}$ & - & Sí & Sí & - & - \\
\hline Servicios amigables para el migrante & Primario & Comunitaria & - & - & - & Sí & - \\
\hline Reforma de salud con cobertura para inmigrantes & $\begin{array}{l}\text { Autoridad sanitaria } \\
\text { Primario }\end{array}$ & $\begin{array}{l}\text { Salud mental } \\
\text { Prevención } \\
\text { Medicamentos }\end{array}$ & - & Sí & Sí & - & - \\
\hline Asistencia médica del estado (Aide Médicale de l'Etat.) & $\begin{array}{l}\text { Primario } \\
\text { Secundario } \\
\text { Terciario }\end{array}$ & $\begin{array}{l}\text { Urgencia } \\
\text { Especialidad } \\
\text { Inmunización } \\
\text { Maternoinfantil }\end{array}$ & - & Sí & Sí & - & - \\
\hline Programa de filipinos en el extranjero & Secundario & - & - & Sí & Sí & - & - \\
\hline Estrategia de diálisis ambulatoria & Secundario & Especialidad & - & Sí & - & - & - \\
\hline Estrategia de diálisis de emergencia & Terciario & Urgencia & - & Sí & - & - & - \\
\hline Código para extranjeros temporalmente presentes & $\begin{array}{l}\text { Primario } \\
\text { Secundario } \\
\text { Terciario }\end{array}$ & - & - & Sí & Sí & - & - \\
\hline Proyecto de Ley SB 741: cobertura de transplante renal a no ciudadanos & Terciario & Especialidad & - & Sí & Sí & - & - \\
\hline $\begin{array}{l}\text { Resolución Conjunta 98: grupo de trabajo legislativo para el estudio de } \\
\text { políticas de transplantes }\end{array}$ & Autoridad sanitaria & - & - & - & - & - & - \\
\hline Real Decreto 16/2012: exclusión de los inmigrantes en situación irregular. & Autoridad sanitaria & - & - & - & - & - & - \\
\hline Política de repatriación médica & Autoridad sanitaria & - & - & - & - & - & - \\
\hline Estrategia binacional de protección social de la salud de los migrantes & $\begin{array}{l}\text { Primario } \\
\text { Secundario } \\
\text { Terciario }\end{array}$ & - & - & - & - & - & - \\
\hline Programa para pacientes no elegibles en centros de salud académicos & $\begin{array}{l}\text { Primario } \\
\text { Terciario }\end{array}$ & $\begin{array}{l}\text { Urgencia } \\
\text { Especialidad }\end{array}$ & - & Sí & Sí & - & - \\
\hline Seguro de salud binacional & $\begin{array}{l}\text { Primario } \\
\text { Terciario }\end{array}$ & $\begin{array}{l}\text { Especialidad } \\
\text { Comunitaria }\end{array}$ & Sí & - & Sí & - & - \\
\hline Seguro obligatorio por el empleador & $\begin{array}{l}\text { Primario } \\
\text { Secundario } \\
\text { Terciario }\end{array}$ & - & - & Sí & Sí & - & - \\
\hline Acceso ampliado a clínicas comunitarias & Primario & $\begin{array}{l}\text { Prevención } \\
\text { Promoción }\end{array}$ & Sí & Sí & - & - & - \\
\hline Derecho a atención de urgencia gratuita & Terciario & Urgencia & - & Sí & Sí & - & Sí \\
\hline Derecho a servicios de emergencia & Terciario & Urgencia & - & Sí & - & - & - \\
\hline Sistema de reembolso específico de la atención médicamente necesaria & $\begin{array}{l}\text { Primario } \\
\text { Secundario } \\
\text { Terciario }\end{array}$ & $\begin{array}{l}\text { Urgencia } \\
\text { Salud mental } \\
\text { Maternoinfantil } \\
\text { Dental } \\
\text { Chequeo médico }\end{array}$ & - & Sí & - & - & - \\
\hline San Fracisco Healthy Kids & $\begin{array}{l}\text { Primario } \\
\text { Secundario } \\
\text { Terciario }\end{array}$ & - & - & Sí & - & - & - \\
\hline Healthy San Francisco & Primario & $\begin{array}{l}\text { Prevención } \\
\text { Chequeo médico }\end{array}$ & - & Sí & Sí & - & - \\
\hline Acceso a servicios de emergencias & Terciario & Urgencia & - & - & - & - & Sí \\
\hline $\begin{array}{l}\text { Cuidado de enfermedad renal en estado terminal para inmigrantes } \\
\text { indocumentados }\end{array}$ & Terciario & $\begin{array}{l}\text { Urgencia } \\
\text { Especialidad }\end{array}$ & - & Sí & - & - & - \\
\hline Unidades de búsqueda, trauma y rescate de la patrulla fronteriza & Primario & Urgencia & Sí & - & - & - & Sí \\
\hline Iniciativas para la salud de los niños (CHI): programa niños saludables & $\begin{array}{l}\text { Secundario } \\
\text { Terciario }\end{array}$ & $\begin{array}{l}\text { Urgencia } \\
\text { Especialidad } \\
\text { Salud mental } \\
\text { Dental } \\
\text { Chequeo médico }\end{array}$ & - & Sí & Sí & - & - \\
\hline Ley de reembolso de salud de inmigrantes & Terciario & Urgencia & - & Sí & Sí & - & - \\
\hline Eliminación del financiamiento público de la atención prenatal & Autoridad sanitaria & - & - & - & - & - & - \\
\hline
\end{tabular}

G: Geográfica; A: Administrativa; F: Financiera; CC: Competencia cultural; CM: Control migratorio 
reembolso al tratamiento de los MISI, bajo argumentos como la integridad financiera del hospital y la continuidad de atención ${ }^{(35)}$. Otra iniciativa es una estrategia binacional de protección de mexicanos en Estados Unidos para aumentar la accesibilidad a los servicios, la cual es apoyada por actores gubernamentales y no gubernamentales, pero que, presenta barreras como la escasa disponibilidad de servicios en el país receptor, la accesibilidad administrativa a servicios públicos y el desincentivo a la búsqueda de servicios en el país de origen por el riesgo de la deportación ${ }^{(22,36)}$.

\section{CURSO DE VIDA}

Seis iniciativas están dirigidas a un segmento etario específico. Dos articulan acciones para niños, niñas y adolescentes. Las coaliciones comunitarias Children's Health Initiatives creadas en California para ampliar el alcance y diseñar nuevos programas de seguro para cubrir a niños que no cumplían con los criterios de elegibilidad, dieron origen a los programas de cobertura Healthy Kids y California Kids, dirigidos a niños no elegibles para cobertura pública por su estatus migratorio o nivel de ingreso familiar (37). Asimismo, en San Francisco fue creado San Francisco Healthy Kids, que ofrece planes de salud subsidiados para residentes de 0 a 18 años no elegibles por otras formas de cobertura ${ }^{(29)}$.

Cuatro iniciativas se dirigen a la población adulta. El programa Health Safety Net de Massachusetts brinda cobertura de servicios preventivos y algunos servicios especializados a adultos no asegurados con bajos ingresos y con cualquier estatus de ciudadanía ${ }^{(38)}$. Otra iniciativa es la Ley 741 del Senado de Illinois, que revisó el programa Medicaid para proporcionar cobertura de trasplante renal a adultos no ciudadanos de bajos ingresos que reciben diálisis ${ }^{(39)}$. Por otra parte, el programa Healthy San Francisco, implementado en la ciudad de San Francisco, brinda acceso universal a la atención médica primaria a todo adulto residente de 18 a 65 años carente de recursos y que no califica para otras formas de cobertura ${ }^{(29)}$.

\section{CONCLUSIONES}

Las 31 iniciativas identificadas en esta revisión dan cuenta de la existencia de respuestas organizadas en el mundo. En términos de disponibilidad, los principales esfuerzos se han orientado a garantizar la atención de emergencia y urgencia a la población MISI. Además, diversas iniciativas han resuelto problemas de accesibilidad administrativa, por medio de normas o mecanismos que permiten atender y/o inscribir a la población MISI en el sistema de salud; así como resolver problemas de accesibilidad financiera.

Un importante número de iniciativas brindan acceso a servicios de nivel primario, lo que es relevante, ya que se ha evidenciado que los MISI tienen menores tasas de utilización de estos servicios que los migrantes regularizados (40). No obstante, no se encontraron iniciativas que facilitaran servicios de rehabilitación, ni que declararan tener perspectiva de género. Esto resulta preocupante ya que muchos estudios muestran la relevancia de desarrollar acciones en salud con perspectiva de género, que contemplen la percepción y prácticas de las mujeres migrantes ${ }^{(41)}$. Preocupa además la presencia de solo una iniciativa relacionada con la competencia intercultural en salud, dados los grandes desafíos relacionados a lo cultural y lo lingüístico que enfrentan tanto migrantes como proveedores ${ }^{(42)}$.

El presente estudió tiene entre sus principales fortalezas haber realizado una exhaustiva revisión de literatura en seis bases de datos científicas que permitieron aumentar el alcance de la búsqueda, junto con permitir a priorila inclusión de cualquier tipo de estudio que describiera una iniciativa. Sin embargo, la heterogeneidad de estudios representó una limitación para encontrar un instrumento estandarizado para la evaluación de la calidad de los estudios seleccionados. Otra limitación fue la ausencia de búsqueda de fuentes de literatura gris relevantes en materia de documentación de diseño, formulación e implementación de políticas públicas e iniciativas gubernamentales y no gubernamentales, como leyes, reportes técnicos y documentos institucionales, entre otros. Finalmente, otra limitación corresponde al período de tiempo en que se realizó la búsqueda, lo que deja fuera a estudios publicados en los últimos dos años, que entregan información que permitiría complementar el análisis realizado, como iniciativas implementadas en Francia ${ }^{(43,44)}$, Italia ${ }^{(43,45)}$, Tailandia ${ }^{(46,47)}$ y Estados Unidos ${ }^{(46)}$, así como también aportan información sobre iniciativas no analizadas en esta revisión, existentes en Noruega ${ }^{(46)}$, Israel ${ }^{(48)}$ y Alemania ${ }^{(49)}$.

De acuerdo con la Organización Mundial de la Salud, garantizar al menos un nivel básico de protección pública de salud a la población MISI es una obligación, desde una perspectiva de salud pública como desde el marco internacional de derechos humanos (50). Adoptar un enfoque de salud pública para la población migrante significa implementar políticas, estrategias y acciones que permitan evitar desigualdades sanitarias entre la población migrante y la población local, eliminar la estigmatización, la discriminación y las barreras de acceso a prestaciones sanitarias, reducir la mortalidad y morbilidad, y minimizar el impacto sanitario negativo relacionado al proceso migratorio ${ }^{(51)}$. En ese sentido, la presente revisión resulta relevante para la salud pública, ya que ofrece una serie de opciones para brindar acceso a los servicios de salud a la población MISI, a fin de reducir sus desigualdades y resultados negativos en salud.

Asimismo, esta revisión visibiliza el déficit de evidencia existente sobre iniciativas que aborden dimensiones de 
Tabla 4. Iniciativas que abordan la problemática de acceso y uso de servicios de salud que enfrentan migrantes internacionales en situación irregular descritas según variables de análisis asociadas a las características de la población

\begin{tabular}{|c|c|c|c|c|}
\hline \multirow[b]{2}{*}{ Iniciativas $(n=31)$} & \multicolumn{4}{|c|}{ Características de la población } \\
\hline & $\begin{array}{l}\text { Etapa del proceso } \\
\text { migratorio }\end{array}$ & $\begin{array}{l}\text { Etapa del } \\
\text { ciclo vital }\end{array}$ & Género & $\begin{array}{l}\text { Sistemas de } \\
\text { creencias }\end{array}$ \\
\hline Protección del riesgo financiero & Destino & - & - & - \\
\hline Servicios amigables para el migrante & Destino & - & - & - \\
\hline Reforma de salud con cobertura para inmigrantes & Destino & - & - & - \\
\hline Asistencia médica del estado (Aide Médicale de l'Etat.) & Destino & - & - & - \\
\hline Programa de filipinos en el extranjero & Destino & - & - & - \\
\hline Estrategia de diálisis ambulatoria & Destino & - & - & - \\
\hline Estrategia de diálisis de emergencia & Destino & - & - & - \\
\hline Programa de asistencia a la tuberculosis de Estambul & Destino & - & - & - \\
\hline Código para extranjeros temporalmente presentes & Destino & - & - & - \\
\hline Proyecto de Ley SB 741: cobertura de transplante renal a no ciudadanos & Destino & - & - & - \\
\hline $\begin{array}{l}\text { Resolución Conjunta 98: grupo de trabajo legislativo para el estudio de } \\
\text { políticas de transplantes }\end{array}$ & Destino & - & - & - \\
\hline $\begin{array}{l}\text { Real Decreto 16/2012: exclusión de los inmigrantes en situación } \\
\text { irregular. }\end{array}$ & Destino & - & - & - \\
\hline Política de repatriación médica & Destino-Retorno & - & - & - \\
\hline Estrategia binacional de protección social de la salud de los migrantes & Destino-Retorno & - & - & - \\
\hline Programa para pacientes no elegibles en centros de salud académicos & Destino & - & - & - \\
\hline $\begin{array}{l}\text { Ley de protección al paciente y cuidado de salud asequible PPACA } \\
\text { (Acceso exclusivo a atención de emergencia) }\end{array}$ & Destino & - & - & - \\
\hline $\begin{array}{l}\text { Ley de reconciliación de responsabilidad personal y oportunidad } \\
\text { (PRWORA) }\end{array}$ & Destino & - & - & - \\
\hline Seguro de salud binacional & $\begin{array}{l}\text { Origen-Destino } \\
\text { Retorno }\end{array}$ & - & - & - \\
\hline Seguro obligatorio por el empleador & Destino & - & - & - \\
\hline Acceso ampliado a clínicas comunitarias & Destino & - & - & - \\
\hline Derecho a atención de urgencia gratuita & Destino & $\begin{array}{l}\text { Niños y niñas } \\
\text { Adultos }\end{array}$ & - & - \\
\hline Derecho a servicios de emergencia & Destino & - & - & - \\
\hline Sistema de reembolso específico de la atención médicamente necesaria & Destino & - & - & - \\
\hline San Fracisco Healthy Kids & Destino & Niños y niñas & - & - \\
\hline Healthy San Francisco & Destino & Adultos & - & - \\
\hline Acceso a servicios de emergencias & Destino & - & - & - \\
\hline $\begin{array}{l}\text { Cuidado de enfermedad renal en estado terminal para inmigrantes } \\
\text { indocumentados }\end{array}$ & Destino & - & - & - \\
\hline Unidades de búsqueda, trauma y rescate de la patrulla fronteriza & Tránsito & - & - & - \\
\hline Iniciativas para la salud de los niños $(\mathrm{CHI})$ : programa niños saludables & Destino & $\begin{array}{l}\text { Niños y niñas } \\
\text { Adolescentes }\end{array}$ & - & - \\
\hline Ley de reembolso de salud de inmigrantes & Destino & - & - & - \\
\hline Eliminación del financiamiento público de la atención prenatal & Destino & - & - & - \\
\hline
\end{tabular}

acceso a los servicios de salud de la población MISI tales como aceptabilidad, contacto y calidad, así como sistemas de creencia y género. Estudios futuros deberían ser capaces de abordar dichas dimensiones y orientar el diseño e implementación de acciones integrales. Es importante también que próximos estudios revisen la literatura gris, puesto que la mayoría de la evidencia relacionada a políticas públicas no está disponible en bases de datos científicas. Esto permitiría tener un mejor conocimiento de las iniciativas que se han implementado en el mundo.
Contribuciones de los autores: DLR y BCV han participado en la concepción del artículo, la recolección de datos, el análisis e interpretación de datos, su redacción y aprobación de la versión final.

Fuentes de financiamiento: Este estudio no contó con fuentes de financiamiento.

Conflictos de interés: Los autores declaran no presentar conflictos de interés.

Material suplementario: Disponible en la versión electrónica de la RPMESP. 


\section{REFERENCIAS BIBLIOGRÁFICAS}

1. United Nations. International Migration Report 2017: Highlights [Internet]. New York: Department of Economic and Social Affairs, Population Division; 2017. [citado 11 octubre de 2018]. Disponible en: http://www.un.org/en/ development/desa/population/migration/ publications/migrationreport/docs/ MigrationReport2017_Highlights.pdf

2. Organización Internacional para las Migraciones. Informe sobre las migraciones en el mundo 2018 [Internet]. Ginebra: OIM; 2018. [citado 11 octubre de 2018]. Disponible en: https://publications.iom. int/system/files/pdf/wmr_2018_sp.pdf

3. Size and development of irregular migration to the EU. Clandestino Research Project. [Internet]. Brussels: European Comission; 2009. [citado 11 octubrede2018].Disponibleen:http:// irregular-migration.net/fileadmin/ irregular-migration/dateien/4. Background_Information/4.2.Policy_ Briefs_EN/ComparativePolicyBrief_ Size Of Irregular Migration Clandestino_Nov09_2.pdf

4. Organización de los Estados Americanos. Anexo 1 - El grado de migración irregular en las Américas. Washington: OEA, 2014. [citado 11 de octubre de 2018]. Disponible en: http://www.migracionoea. org/index.php/es/sicremi-es/45-sicremi/ publicacion-2014/informe-parte-iesp/379-annex-1-the-extent-of-irregularmigration-in-the-americas-2.html

5. Comisión Interamericana de Derechos Humanos. Derechos humanos de migrantes, refugiados, apátridas, víctimas de trata de personas y desplazados internos: Normas y Estándares del Sistema Interamericano de Derechos Humanos [Internet]. Washington: CIDH; 2015. [citado 11 octubre de 2018]. Disponible en: http://www.oas.org/es/cidh/informes/ pdfs/MovilidadHumana.pdf

6. Betts A. Soft Law and the Protection of Vulnerable Migrants. Georget Immigr Law J. 2010;24:533-52.

7. Simich L. Status and Health Security: An Exploratory Study of Irregular Immigrants in Toronto. Can J Public Health. 2007;98(5):369-73.

8. Collantes S. Access to health care for undocumented migrants and asylum seekers in $10 \mathrm{EU}$ countries: law and practice. Paris: HUMA Network; 2009.
9. Naciones Unidas. Convención internacional sobre la protección de los derechos de todos los trabajadores migratorios y de sus familiares. En: Los Principales Tratados Internacionales de Derechos Humanos [Internet]. Naciones Unidas; 2014 [citado 22 de septiembre de 2018]. p. 219-69. Disponible en: http://www.un-ilibrary. org/human-rights-and-refugees/losprincipales-tratados-internacionales-dederechos-humanos_f8d551dd-es

10. Consejo Económico y Social de las Naciones Unidas. El derecho al disfrute del más alto nivel posible de salud [Internet]. New York: Naciones Unidas; 2000. [citado 11 octubre de 2018]. Disponible en: http://www.acnur.org/fileadmin/ Documentos/BDL/2001/1451.pdf?view

11. Terraza-Núñez R, Toledo $\mathrm{D}$, Vargas I, Vázquez ML. Perception of the Ecuadorian population living in Barcelona regarding access to health services. Int $\mathrm{J}$ Public Health. 2010;55(5):381-90. doi: 10.1007/s00038-010-0180-1.

12. Priebe S, Giacco D, El-Nagib R. Public health aspects of mental health among migrants and refugees: a review of the evidence on mental health care for refugees, asylum seekers and irregular migrants in the WHO European Region. [Internet]. Copenhagen: World Health Organization; 2016. [citado 11 octubre de 2018]. Disponible en: https://www. ncbi.nlm.nih.gov/pubmedhealth/ PMH0096089/pdf/PubMedHealth_ PMH0096089.pdf

13. Dorn T, Ceelen M, Tang M-J, Browne JL, de Keijzer KJ, Buster MC, et al. Health care seeking among detained undocumented migrants: a cross-sectional study. BMC Public Health. 2011;11(1):1. doi: 10.1186/1471-2458-11-190.

14. Blignault I, Ponzio V, Ye Rong, Eisenbruch M. A Qualitative Study of Barriers to Mental Health Services Utilisation Among Migrants From Mainland China in South-East Sydney. Int J Soc Psychiatry. 2008;54(2):180-90. doi: 10.1177/0020764007085872.

15. Liberona Concha N. Poder, contrapoder $\mathrm{y}$ relaciones de complicidad entre inmigrantes sudamericanos y funcionarios del sistema público de salud chileno. Si Somos Am. 2015;15(2):15-40. doi: 10.4067/S0719-09482015000200002.

16. Cabieses B, Flaño J, Gálvez P, Ajraz N. Escenario actual de la vida y salud de migrantes en la comuna de Recoleta. Región del Bío Bío: Universidad del Desarrollo; 2017.
17. Aizenberg L, Rodríguez ML, Carbonetti A. Percepciones de los equipos de salud en torno a las mujeres migrantes bolivianas y peruanas en la ciudad de Córdoba. Migr Int. 2015;8(1):65-94.

18. Naciones Unidas, CEPAL. Consenso de Montevideo sobre población y desarrollo. Primera reunión de la Conferencia Regional sobre Población y Desarrollo de América Latina y el Caribe. [Internet]. Montevideo:CEPAL;2013. [citado 11 octubre de 2018]. Disponible en: http:// repositorio.cepal.org/handle/11362/21835

19. European Parliament [Internet]. Brussels: EP; 2019. European Parliament resolution of 8 March 2011 on reducing health inequalities in the EU (2010/2089(INI)). [citado 11 octubre de 2018]. Disponible en: http://www.europarl. europa.eu/sides/getDoc.do?pubRef=-// $\mathrm{EP} / / \mathrm{TEXT}+\mathrm{TA}+\mathrm{P} 7-\mathrm{TA}-2011$ $0081+0+\mathrm{DOC}+\mathrm{XML}+\mathrm{V} 0 / / \mathrm{EN}$

20. United Nation Statistics Division New York: UNSD; 2019. [Internet] Methodology: Standard country or area codes for statistical use (M49) [citado 11 octubre de 2018]. Disponible en: https:// unstats.un.org/unsd/methodology/m49/

21. Yasin Y, Biehl K, Erol M. Infection of the Invisible: Impressions of a Tuberculosis Intervention Program for Migrants in Istanbul. J Immigr Minor Health. 2015;17(5):1481-6. doi: 10.1007/ s10903-014-0115-7.

22. Wallace SP, Rodriguez M, Padilla-Frausto I, Arredondo A, Orozco E. Improving access to health care for undocumented immigrants in the United States. Salud Pública Mex. 2013;55:s508-s514.

23. Tangcharoensathien V, Thwin AA, Patcharanarumol W. Implementing health insurance for migrants, Thailand. Bull World Health Organ. 2017;95(2):14651. doi: 10.2471/BLT.16.179606.

24. Keim SM, Reiser F, Shetty S, RangerMoore J. Wilderness rescue and border enforcement along the Arizona Mexico border-the Border Patrol Search, Trauma and Rescue Unit. Wilderness Environ Med. 2009;20(1):39-42. doi: 10.1580/08-WEME-OR-245.1.

25. André J-M, Azzedine F. Access to healthcare for undocumented migrants in France: a critical examination of State Medical Assistance. Public Health Rev. 2016;37(1). doi: 10.1186/s40985-0160017-4. 
26. De Vito E, de Waure C, Specchia ML, Parente P, Azzolini E, Frisicale EM, et al. Are undocumented migrants' entitlements and barriers to healthcare a public health challenge for the European Union? Public Health Rev. 2016;37:13. doi: 10.1186/ s40985-016-0026-3.

27. Cacari Stone L, Steimel L, VasquezGuzman E, Kaufman A. The Potential Conflict Between Policy and Ethics in Caring for Undocumented Immigrants at Academic Health Centers: Acad Med. 2014;89(4):536-9. doi: 10.1097/ ACM.0000000000000187.

28. Guinto RLLR, Curran UZ, Suphanchaimat R, Pocock NS. Universal health coverage in 'One ASEAN': are migrants included? Glob Health Action. 2015;8(1):25749. doi: 10.3402/gha.v8.25749.

29. Marrow HB. Deserving to a point: Unauthorized immigrants in San Francisco's universal access healthcare model. Soc Sci Med. 2012;74(6):846-54. doi: 10.1016/j.socscimed.2011.08.001.

30. Biswas D, Toebes B, Hjern A, Ascher H, Norredam M. Access to health care for undocumented migrants from a human rights perspective: a comparative study of Denmark, Sweden, and The Netherlands. Health Hum Rights. 2012;14(2):49-60.

31. Kuiper H, Richwald GA, Rotblatt $H$, Asch S. The communicable disease impact of eliminating publicly funded prenatal care for undocumented immigrants. Matern Child Health J. 1999;3(1):39-52.

32. Edward J. Undocumented Immigrants and Access to Health Care: Making a Case for Policy Reform. Policy Polit Nurs Pract. 2014;15(1-2):5-14. doi: $10.1177 / 1527154414532694$

33. Grit K, den Otter JJ, Spreij A. Access to Health Care for Undocumented Migrants: A Comparative Policy Analysis of England and the Netherlands. J Health Polit Policy Law. 2012;37(1):37-67. doi: 10.1215/03616878-1496011.

34. Van der Laat C. La migración internacional como determinante social de la salud en Chile: evidencia y propuestas para políticas públicas. En: La migración internacional como determinante social de la salud en Chile [Internet]. Región de Bio Bio: Universidad del Desarrollo; 2017. p. 29-38. Disponible en: http://www.udd. cl/dircom/pdfs/Libro_La_migracion_ internacional.pdf

35. Young M, Phil M, Lehmann, L. Undocumented Injustice? Medical
Repatriation and the Ends of Health Care. N Engl J Med. 2014;370(7):669-73. doi: 10.1056/NEJMhle1311198.

36. Arredondo A, Orozco E, Asch $S$, Rodriguez M. Indicadores de gobernanza para el desarrollo de estrategias binacionales de protección social en salud de los migrantes. Rev Saúde E Soc. 2013;22(2):310-27.

37. Stevens GD, Rice K, Cousineau MR. Children's Health Initiatives in California: The Experiences of Local Coalitions Pursuing Universal Coverage for Children. Am J Public Health. 2007;97(4):738-43. doi: 10.2105/AJPH.2006.088690.

38. Joseph TD. What Health Care Reform Means for Immigrants: Comparing the Affordable Care Act and Massachusetts Health Reforms.J Health Polit Policy Law. 2015;3445632. doi: 10.1215/036168783445632.

39. Ansell D, Pallok K, Guzman MD, Flores M, Oberholzer J. Illinois Law Opens Door To Kidney Transplants For Undocumented Immigrants. Health Aff (Millwood). 2015;34(5):781-7. doi: 10.1377/hlthaff.2014.1192.

40. Winters M, Rechel B, de Jong L, Pavlova M. A systematic review on the use of healthcare services by undocumented migrants in Europe. BMC Health Serv Res. 2018;18(1). doi: 10.1186/s12913018-2838-y.

41. Aizenberg L, Maure G. Migración, salud y género: abordajes de proveedores de salud en la atención de mujeres migrantes bolivianas en la provincia de Mendoza, Argentina. REMHU Rev Interdiscip Mobili Humana. 2017;25(51):149-64.doi: 10.1590/198085852503880005110 .

42. Suphanchaimat R, Kantamaturapoj K, Putthasri W, Prakongsai P. Challenges in the provision of healthcare services for migrants: a systematic review through providers' lens. BMC Health Serv Res. junio de 2015;15(1). doi: 10.1186/ s12913-015-1065-z.

43. Santilli C. Medical Care, Screening and Regularization of Sub-Saharan Irregular Migrants Affected by Hepatitis B in France and Italy. J Immigr Minor Health. 2018;20(3):668-73. doi: 10.1007/ s10903-017-0585-5.

44. Vignier N, Desgrées du Loû A, Pannetier J, Ravalihasy A, Gosselin A, Lert F, et al. Access to health insurance coverage among sub-Saharan African migrants living in France: Results of the ANRSPARCOURS study. Gusmano M, editor.
Plos One. 2018;13(2):e0192916. doi: 10.1371/journal.pone.0192916.

45. Dalla Zuanna T, Del Manso M, Giambi C, Riccardo F, Bella A, Caporali M, et al. Immunization Offer Targeting Migrants: Policies and Practices in Italy. Int J Environ Res Public Health. 2018;15(5):968. doi: 10.3390/ijerph15050968.

46. Onarheim KH, Melberg A, Meier BM, Miljeteig I. Towards universal health coverage: including undocumented migrants. BMJ Glob Health. 2018;3(5):e001031. doi: 10.1136/ bmjgh-2018-001031.

47. Suphanchaimat R, Pudpong N, Prakongsai P, Putthasri W, Hanefeld J, Mills A. The Devil Is in the DetailUnderstanding Divergence between Intention and Implementation of Health Policy for Undocumented Migrants in Thailand. Int J Environ Res Public Health. 2019;16(6):1016. doi: 10.3390/ ijerph16061016.

48. Mor Z, Raveh Y, Lurie I, Leventhal A, Gamzu R, Davidovitch N, et al. Medical condition and care of undocumented migrants in ambulatory clinics in Tel Aviv, Israel: assessing unmet needs. BMC Health Serv Res. 2017;17(1). doi: 10.1186/s12913-017-2421-y.

49. Mylius M, Dreesman J,Zühlke C, Mertens E. Reduce obstacles, promote health-healthcare access for undocumented migrants within a pilot project in Lower Saxony, Germany (2016-2018). Bundesgesundheitsblatt Gesundheitsforschung - Gesundheitsschutz. 2019;62(8):927-34.. doi: 10.1007/s00103 019-02972-4.

50. Organización Mundial dela Salud. Migración internacional, salud y derechos humanos [Internet]. Ginebra: OMS; 2005. [citado 11 octubre de 2018]. Disponible en: http:// www.who.int/hhr/activities/2005\%20 PRT\%2016325\%20ADD\%201\%20Migr_ HHR-Spanish\%20edition.pdf

51. Organización Mundial de la Salud. Salud de los migrantes: Informe de la Secretaría. [Internet]. Ginebra: OMS; 2008. [citado 11 octubre de 2018]. Disponible en: http://apps.who.int/iris/bitstream/ handle/10665/26222/A61_12-sp.pd$\mathrm{f} ;$ jsessionid $=7 \mathrm{~F} 214663 \mathrm{CBE} 47973 \mathrm{~F}-$ $29207796264 \mathrm{~F} 15 \mathrm{~B}$ ?sequence $=1$

Correspondencia: Daniel Larenas Rosa

Dirección: Av. Independencia 1027, Independencia, Santiago, Chile

Teléfono: +562 29786181

Correo electrónico:d_larenas@ug.uchile.cl 